\title{
Using Full Information When Computing Modes of Post-Newtonian Waveforms From Inspiralling Compact Binaries in Circular Orbit
}

\author{
Lawrence E. Kidder \\ Center for Radiophysics and Space Research, Cornell University, Ithaca, New York, 1485 团
}

(Dated: October 31, 2018)

\begin{abstract}
The increasing sophistication and accuracy of numerical simulations of compact binaries (especially binary black holes) presents the opportunity to test the regime in which post-Newtonian (PN) predictions for the emitted gravitational waves are accurate. In order to confront numerical results with those of post-Newtonian theory, it is convenient to compare multipolar decompositions of the two waveforms. It is pointed out here that the individual modes can be computed to higher post-Newtonian order by examining the radiative multipole moments of the system, rather than by decomposing the $2.5 \mathrm{PN}$ polarization waveforms. In particular, the dominant $(l=2, m= \pm 2)$ mode can be computed to $3 P N$ order. Individual modes are computed to as high a post-Newtonian order as possible given previous post-Newtonian results.
\end{abstract}

PACS numbers: $04.25 . \mathrm{Nx}, 04.25 . \mathrm{Dm}$

\section{INTRODUCTION}

The first generation of laser interferometric gravitational wave detectors is now operating at or near their design sensitivities [1, 2, 3, 4, 5]. One of the most promising sources that they may detect are the inspiral and merger of compact binaries systems with black holes or neutron stars. One of the primary means of detecting and interpreting the signals from such systems will be the use of a matched filtering technique, which requires accurate templates with which to match a theoretical model to a noisy signal. Until the last several orbits prior to merger, the post-Newtonian $(\mathrm{PN})$ approximation is expected to be sufficient to generate accurate templates, while numerical relativity will be needed to help construct accurate templates covering the late inspiral and merger phases.

Estimates of the accuracy of post-Newtonian templates and the effect of this accuracy on detecting gravitational waves have led the post-Newtonian expansion to be pushed to high order $[6,67,8,9]$. Currently the equations of motion and the gravitational wave energy flux are known through 3.5PN order [10, 11], and the polarization waveform amplitudes are known through 2.5PN order [12, 13]. As the post-Newtonian expansion is a slow-motion, weak-field approximation to general relativity, and the motion is not so slow and the fields not so weak during the last orbits prior to merger, it is legitimate to ask where the post-Newtonian expansion breaks down.

Recent breakthroughs in numerical relativity 14, 15, 16, 17] have finally provided the possibility of testing the validity of the post-Newtonian expansion in the late inspiral. Initial studies focused on qualitative comparisons between post-Newtonian generated waveforms and numerical simulations of the final two to four orbits prior to merger [18, 19, 20]. More recently, attempts have been

*Electronic address: kidder@astro.cornell.edu made to quantify the difference between post-Newtonian generated waveforms and numerical simulations of a nonspinning equal-mass binary inspiral lasting more than eight orbits [21, 22, 23]. As long inspiral simulations are computationally expensive to perform, it is important to quantify the errors in the post-Newtonian generated waveforms and determine how they influence the detection of gravitational waves. One would also like to know the accuracy with which the parameters such as the individual masses and spins of the binary can be determined from the observed waveform.

One of the principal ways of quantifying the accuracy of the post-Newtonian approximation is to compare the amplitude and phase of the gravitational waveform with that from numerical simulations. Unlike gravitational wave detection which is sensitive only to the waveform in one particular direction, comparisons between numerical simulations and post-Newtonian waveforms can be performed over the entire sphere. Therefore it is convenient to project the waveforms onto spin-weighted spherical harmonics and compare the individual components. Previous studies [18, 19], however, have computed the spinweighted spherical harmonic components of the postNewtonian amplitude in such a way that has not made full use of current post-Newtonian results.

This situation has arisen primarily because of how the post-Newtonian gravitational wave amplitude is presented. Often the final result of the post-Newtonian computation of the waveform is given as the polarization waveforms as a function of the direction to the observer (as this is most useful when constructing templates). Currently the polarization waveforms are known to $2.5 \mathrm{PN}$ order in amplitude [12, 13]. In previous comparisons between post-Newtonian and numerical waveforms, it was the polarization waveforms that were projected into spin-weighted spherical harmonics in order to determine the post-Newtonian components. The post-Newtonian polarization waveforms are computed by truncating an infinite sum of radiative multipole moments at a given post-Newtonian order 24]. As will 
be demonstrated in this paper, it makes more sense to project the waveform onto spin-weighted spherical harmonics prior to truncating the series as this retains the full information currently known. In particular, the dominant $(l=2, m=2)$ component of the waveform can be computed to $3 P N$ order.

While knowledge of a particular spin-weighted components of a gravitational waveform is of limited value for computing templates for detection and characterization of gravitational waves, it can be used to quantify the difference between post-Newtonian and numerical waveforms. In addition, if the $3 \mathrm{PN}$ correction to the amplitude of the $(l=2, m=2)$ component significantly improves the agreement between the post-Newtonian and numerical waveforms, this would suggest it might be worth the effort needed to compute the polarization waveforms to $3 \mathrm{PN}$ order. It has been shown that higher post-Newtonian corrections in the amplitude can improve detection rates 25, 26, 27] as well as improve parameter extraction [28, 29, 30, 31, 32, 33, 34].

The relationship between the gravitational waveform and radiative multipole moments is reviewed in Sec. II] It is pointed out that the the spin-weighted spherical harmonic components of the waveform can be computed directly from the radiative mulitpole moments of the system in Sec. IIB. Current post-Newtonian results that affect the computation of the radiative multipole moments are summarized in Sec. III. The radiative multipole moments are computed to as high a post-Newtonian order as possible in Sec. IV. Conclusions are presented in Sec. V.

\section{GRAVITATIONAL WAVEFORM AND SPIN-WEIGHTED SPHERICAL HARMONICS}

\section{A. Notation}

Let $X^{\mu}=(c T, X, Y, Z)$ be coordinates in an asymptotically flat radiative coordinate system, with $\vec{e}_{T}$ the unit timelike normal, and $\left(\vec{e}_{X}, \vec{e}_{Y}, \vec{e}_{Z}\right)$ the spatial orthonormal coordinate basis vectors. Let $(c T, R, \Theta, \Phi)$ be the corresponding spherical coordinate system with corresponding basis vectors $\left(\vec{e}_{R}, \vec{e}_{\Theta}, \vec{e}_{\Phi}\right)$. Let $T_{R}=T-R / c$ denote retarded time in radiative coordinates.

Greek letters are used for spacetime indices, Latin letters for spatial indices. As indices can be raised or lowered using the Minkowski metric, components of tensors will typically have down indices. Let $N_{i}$ be a component of the unit radial vector $\vec{e}_{R}$. A capital letter for an index denotes a multi-index (i.e. $T_{L}=T_{i_{1} i_{2} \cdots i_{\ell}}$ ). For a vector $V$, let $V_{L}$ be a product of components of the vector (i.e. $\left.V_{L}=V_{i_{1}} V_{i_{2}} \cdots V_{i_{\ell}}\right)$. Repeated spatial indices (including multi-indices) are to be summed over. Tensors that are fully symmetric and trace-free (STF) will be denoted with capital script letters (e.g. $\mathcal{I}_{i j}$ ). Symmetrization, anti-symmetrization, and STF projection of indices are denoted by (), [], and $<>$ respectively, with underlined indices between the delimiters to be excluded from the operation (e.g. $\left.2 T_{(a \underline{b} c)}=T_{a b c}+T_{c b a}\right)$.

Let $(\vec{k}, \vec{l}, \vec{m})$ be a set of null vectors defined by:

$$
\begin{aligned}
\vec{k} & =\frac{1}{\sqrt{2}}\left(\vec{e}_{T}+\vec{e}_{R}\right), \\
\vec{l} & =\frac{1}{\sqrt{2}}\left(\vec{e}_{T}-\vec{e}_{R}\right), \\
\vec{m} & =\frac{1}{\sqrt{2}}\left(\vec{e}_{\Theta}+i \vec{e}_{\Phi}\right) .
\end{aligned}
$$

The spin-weighted spherical harmonics are defined in terms of the Wigner $d$-functions by

$$
{ }_{-s} Y^{\ell m}(\Theta, \Phi)=(-1) s \sqrt{\frac{2 \ell+1}{4 \pi}} d_{m s}^{\ell}(\Theta) e^{i m \Phi},
$$

where

$$
\begin{aligned}
& d_{m s}^{\ell}(\Theta)=\sqrt{(\ell+m) !(\ell-m) !(\ell+s) !(\ell-s) !} \\
& \times \sum_{k=k_{i}}^{k_{f}} \frac{(-1)^{k}\left(\sin \frac{\Theta}{2}\right)^{2 k+s-m}\left(\cos \frac{\Theta}{2}\right)^{2 \ell+m-s-2 k}}{k !(\ell+m-k) !(\ell-s-k) !(s-m+k) !}
\end{aligned}
$$

where $k_{i}=\max (0, m-s)$ and $k_{f}=\min (\ell+m, \ell-s)$.

\section{B. Polarization Waveforms and Modes}

The asymptotic waveform $h_{i j}^{T T}$ can be decomposed into two sets of symmetric trace-free (STF) radiative multipole moments as [24]

$$
\begin{aligned}
h_{i j}^{T T}= & \frac{4 G}{c^{2} R} \Pi_{i j m n} \sum_{\ell=2}^{\infty}\left\{\frac{1}{c^{\ell} \ell !} \mathcal{U}_{m n L-2}\left(T_{R}\right) N_{L-2}\right. \\
& \left.+\frac{2 \ell}{c^{\ell+1}(\ell+1) !} \epsilon_{p q(m} \mathcal{V}_{n) p L-2}\left(T_{R}\right) N_{q L-2}\right\} .
\end{aligned}
$$

Here $\mathcal{U}_{L}\left(T_{R}\right)$ are the mass-type moments and $\mathcal{V}_{L}\left(T_{R}\right)$ are the current-type moments. In Sec. IIIA these radiative multipole moments will be related to multipole moments describing the source in the near-zone. Note that higher multipole moments contribute to the waveform at higher post-Newtonian order, so that at any finite post-Newtonian order, only a finite number of multipoles contribute. The transverse-traceless (TT) projection operator $\Pi_{i j m n}$ is given by

$$
\Pi_{i j m n}=P_{i m} P_{j n}-\frac{1}{2} P_{i j} P_{m n}
$$

where $P_{i j}=\delta_{i j}-N_{i} N_{j}$.

Given an orthonormal triad $(\vec{N}, \vec{P}, \vec{Q})$, the polarization waveforms can be given by

$$
\begin{aligned}
& h_{+}=\frac{1}{2}\left(P_{m} P_{n}-Q_{m} Q_{n}\right) h_{m n}^{T T}, \\
& h_{\times}=\frac{1}{2}\left(P_{m} Q_{n}+P_{n} Q_{m}\right) h_{m n}^{T T} .
\end{aligned}
$$


A natural (but by no means unique ${ }^{1}$ ) choice for the triad is $\vec{P}=\vec{e}_{\Theta}$ and $\vec{Q}=\vec{e}_{\Phi}$. It is then straightforward to show that

$$
h_{+}-i h_{\times}=m_{m}^{*} m_{n}^{*} h_{m n}^{T T},
$$

where $*$ denotes complex conjugation. It will now be shown how $h_{+}-i h_{\times}$can be decomposed into modes using spin-weighted spherical harmonics of weight -2

$$
h_{+}-i h_{\times}=\sum_{\ell=2}^{\infty} \sum_{m=-\ell}^{\ell} h_{-2}^{\ell m} Y^{\ell m}(\Theta, \Phi) .
$$

An alternative expression for the waveform is given by 24$]$

$$
\begin{aligned}
h_{i j}^{T T}= & \frac{G}{c^{2} R} \sum_{\ell=2}^{\infty} \sum_{m=-\ell}^{\ell}\left\{\frac{1}{c^{\ell}} U^{\ell m}\left(T_{R}\right) T_{i j}^{E 2, \ell m}\right. \\
& \left.+\frac{1}{c^{\ell+1}} V^{\ell m}\left(T_{R}\right) T_{i j}^{B 2, \ell m}\right\},
\end{aligned}
$$

where $T_{i j}^{E 2, \ell m}$ and $T_{i j}^{B 2, \ell m}$ are pure-spin tensor harmonics, and where the mass multipole moments $U^{\ell m}\left(T_{R}\right)$ and current multipole moments $V^{\ell m}\left(T_{R}\right)$ are related to their STF counterparts by 24]

$$
\begin{aligned}
U^{\ell m} & =\frac{16 \pi}{(2 \ell+1) ! !} \sqrt{\frac{(\ell+1)(\ell+2)}{2 \ell(\ell-1)}} \mathcal{U}_{L} \mathcal{Y}_{L}^{\ell m *}, \\
V^{\ell m} & =\frac{-32 \pi \ell}{(2 \ell+1) ! !} \sqrt{\frac{(\ell+2)}{2 \ell(\ell+1)(\ell-1)}} \mathcal{V}_{L} \mathcal{Y}_{L}^{\ell m *},
\end{aligned}
$$

where $\mathcal{Y}_{L}^{\ell m}$ are the STF spherical harmonics which are related to the scalar spherical harmonics by

$$
Y^{\ell m}(\Theta, \Phi)=\mathcal{Y}_{L}^{\ell m} N_{L}
$$

The pure-spin tensor harmonics are related to the spinweighted spherical harmonics by [24]

$$
\begin{aligned}
T_{i j}^{E 2, \ell m} & =\frac{1}{\sqrt{2}}\left({ }_{-2} Y^{\ell m} m_{i} m_{j}+{ }_{2} Y^{\ell m} m_{i}^{*} m_{j}^{*}\right), \\
T_{i j}^{B 2, \ell m} & =\frac{-i}{\sqrt{2}}\left({ }_{-2} Y^{\ell m} m_{i} m_{j}-{ }_{2} Y^{\ell m} m_{i}^{*} m_{j}^{*}\right) .
\end{aligned}
$$

Combining Eqs. (10), (12), and (16) yields

$$
\begin{aligned}
h_{+}-i h_{\times}= & \frac{G}{c^{2} R} \sum_{\ell=2}^{\infty} \sum_{m=-\ell}^{\ell}\left\{\frac{1}{c^{\ell} \sqrt{2}} U^{\ell m}\left(T_{R}\right)\right. \\
& \left.-\frac{i}{c^{\ell+1} \sqrt{2}} V^{\ell m}\left(T_{R}\right)\right\}-2 Y^{\ell m}(\Theta, \Phi)
\end{aligned}
$$

\footnotetext{
${ }^{1}$ The 2.5PN polarization waveforms in [12] are defined with $\vec{P}=$ $-\vec{e}_{\Phi}$ and $\vec{Q}=\vec{e}_{\Theta}$ evaluated at $\Theta=i, \Phi=\pi / 2$.
}

Thus the spin-weighted spherical harmonic components of the waveform are given by

$$
h^{\ell m}=\frac{G}{\sqrt{2} R c^{\ell+2}}\left(U^{\ell m}\left(T_{R}\right)-\frac{i}{c} V^{\ell m}\left(T_{R}\right)\right) .
$$

Note that the spin-weighted spherical harmonic components can be computed directly from the radiative multipole moments. It is not necessary to compute the waveform as a function of position, and then project out the components as is commonly done in the recent literature. In fact, not only are these extra steps unnecessary, they cause individual components to be truncated at a lower post-Newtonian order than they need to be. In particular, the dominant $h^{22}$ component is truncated to $2.5 \mathrm{PN}$ order, when it can be computed (as will be shown below) to $3 \mathrm{PN}$ order directly from the radiative mass quadrupole moment.

This situation arises as the complete polarization waveforms $h_{+}$and $h_{\times}$are known only through 2.5PN order. But as discussed in [12], the computation of the $3 \mathrm{PN}$ waveform is not limited by the post-Newtonian order of the dominant mass quadrupole contribution, but by the order of higher multipoles. Therefore to obtain the spin-weighted spherical harmonic components of the waveform to as high a post-Newtonian order as possible, they should be computed directly from the radiative multipole moments. As discussed in Sec. $\mathrm{V}$ below, this will allow post-Newtonian results to be probed to a higher order when comparing with numerical simulations. Table I summarizes the post-Newtonian order to which each radiative multipole moment needs to computed to obtain the full 2.5PN order polarization waveforms, and the post-Newtonian order to which they are currently known.

\section{SUMMARY OF CURRENT POST-NEWTONIAN RESULTS}

The post-Newtonian approximation is a slow-motion, weak-field approximation to general relativity with an expansion parameter $\epsilon \sim(v / c)^{2} \sim\left(G m / r c^{2}\right)$. For a binary system of two point masses $m_{1}$ and $m_{2}, v$ is the magnitude of the relative velocity, $m=m_{1}+m_{2}$, and $r$ is the separation. In order to produce a postNewtonian waveform, it is necessary to solve both the post-Newtonian equations of motion describing the binary, and the post-Newtonian equations describing the generation of gravitational waves. For a complete review of post-Newtonian methods applied to inspiralling compact binaries, see 35].

\section{A. Post-Newtonian Computation of Radiative Multipoles}

In Sec. II B the spin-weighted spherical harmonic components have been related to the radiative multipole mo- 
TABLE I: The post-Newtonian order to which each radiative multipole moment must be computed to obtain the full $2.5 \mathrm{PN}$ polarization waveforms $h_{+}$and $h_{\times}$, and the order to which they are known.

\begin{tabular}{ccc}
\hline \hline Radiative Multipole & PN order for $h_{+, \times}$ & Known PN order \\
$\mathcal{I}_{i j}$ & 2.5 & 3 \\
$\mathcal{J}_{i j}$ & 2 & 2 \\
$\mathcal{I}_{i j k}$ & 2 & 2 \\
$\mathcal{J}_{i j k}$ & 1.5 & $1.5^{a}$ \\
$\mathcal{I}_{i j k l}$ & 1.5 & 2 \\
$\mathcal{J}_{i j k l}$ & 1 & 1 \\
$\mathcal{I}_{i j k l m}$ & 1 & 1 \\
$\mathcal{J}_{i j k l m}$ & 0.5 & 1 \\
$\mathcal{I}_{i j k l m n}$ & 0.5 & 1 \\
$\mathcal{J}_{i j k l m n}$ & 0 & 1 \\
$\mathcal{I}_{i j k l m n o}$ & 0 & 1 \\
$\mathcal{I}_{L}(\ell>7)$ & - & 1 \\
$\mathcal{J}_{L}(\ell>6)$ & - & 1 \\
\hline \hline
\end{tabular}

${ }^{a}$ The radiative current octupole could be computed to $2 \mathrm{PN}$ order by a computation of the $2 \mathrm{PN}$ correction to the source current octupole.

ments. In order to use Eq. (19) it is necessary to relate the radiative moments to a description of the compact binary system. The post-Newtonian wave generation formalism has been developed 36, 37, 38, 39, 40, 41, 42 in a systematic manner to relate the radiative multipole moments $\left\{\mathcal{U}_{L}, \mathcal{V}_{L}\right\}$ to a set of six STF source moments $\left\{\mathcal{I}_{L}, \mathcal{J}_{L}, \mathcal{W}_{L}, \mathcal{X}_{L}, \mathcal{Y}_{L}, \mathcal{Z}_{L}\right\}$, which can be computed from the stress-energy pseudo-tensor of the material and gravitational fields of the source. A set of two canonical source moments $\left\{\mathcal{M}_{L}, \mathcal{S}_{L}\right\}$ can be computed as a nonlinear functional of the source moments as an intermediate step between the source moments and the radiative moments. The radiative moments are then given as nonlinear (and even nonlocal) functionals of the canonical moments. It turns out that two of the source moments, the source mass moments $\mathcal{I}_{L}$ and the source current moments $\mathcal{J}_{L}$ are dominant, while the other four parameterize a gauge transformation and only make a contribution to the canonical source moments starting at $2.5 \mathrm{PN}$ order. To the post-Newtonian order needed in this paper, only the canonical mass quadrupole moment contains a correction from its corresponding source moment [43, 44]

$$
\mathcal{M}_{i j}=\mathcal{I}_{i j}+\frac{4 G}{c^{5}}\left(\mathcal{W}^{(2)} \mathcal{I}_{i j}-\mathcal{W}^{(1)} \mathcal{I}_{i j}^{(1)}\right)+O\left(\epsilon^{7 / 2}\right)
$$

where $\mathcal{T}_{L}^{(p)}$ denotes the $p$ th time derivative of $\mathcal{T}_{L}$ and $O\left(\epsilon^{k}\right)$ denotes the $k$ th-order and higher post-Newtonian corrections that are unknown. The other canonical source moments are simply related to the source moments

$$
\begin{aligned}
\mathcal{M}_{L} & =\mathcal{I}_{L}+O\left(\epsilon^{5 / 2}\right), \\
\mathcal{S}_{L} & =\mathcal{J}_{L}+O\left(\epsilon^{5 / 2}\right) .
\end{aligned}
$$

The radiative mass quadrupole is given by

$$
\begin{aligned}
\mathcal{U}_{i j}\left(T_{R}\right)= & \mathcal{M}_{i j}^{(2)}\left(T_{R}\right)+\frac{2 G M}{c^{3}} \int_{0}^{\infty} d \tau \mathcal{M}_{i j}^{(4)}\left(T_{R}-\tau\right)\left[\ln \left(\frac{c \tau}{2 r_{0}}\right)+\frac{11}{12}\right]-\frac{2}{7} \frac{G}{c^{5}} \int_{0}^{\infty} d \tau \mathcal{M}_{a\langle i}^{(3)}\left(T_{R}-\tau\right) \mathcal{M}_{j\rangle a}^{(3)}\left(T_{R}-\tau\right) \\
& +\frac{G}{c^{5}}\left\{\frac{1}{7} \mathcal{M}_{a\langle i}^{(5)} \mathcal{M}_{j\rangle a}-\frac{5}{7} \mathcal{M}_{a\langle i}^{(4)} \mathcal{M}_{j\rangle a}^{(1)}-\frac{2}{7} \mathcal{M}_{a\langle i}^{(3)} \mathcal{M}_{j\rangle a}^{(2)}+\frac{1}{3} \epsilon_{a b\langle i} \mathcal{M}_{j\rangle a}^{(4)} \mathcal{S}_{b}\right\} \\
& +\frac{2 G^{2} M^{2}}{c^{6}} \int_{0}^{\infty} d \tau \mathcal{M}_{i j}^{(5)}\left(T_{R}-\tau\right)\left[\ln ^{2}\left(\frac{c \tau}{2 r_{0}}\right)+\frac{57}{70} \ln \left(\frac{c \tau}{2 r_{0}}\right)+\frac{124627}{44100}\right]+O\left(\epsilon^{7 / 2}\right)
\end{aligned}
$$

The first integral in the above expression is the dominant radiation tail at $1.5 \mathrm{PN}$ order obtained in 38]. The $2.5 \mathrm{PN}$ non-linear memory integral has been obtained in [38, 40, 45, 46, 47]. The other non-linear interactions at $2.5 \mathrm{PN}$ order were obtained in [40]. Finally the $3 \mathrm{PN}$ "tail of tail" integral was derived in [41]. The tail integrals involve nonlinear interactions with the mass monopole $M$ (equivalent to the ADM mass) of the system. The tail integrals also contain a freely-specifiable constant $r_{0}$ that corresponds to the choice of the origin of radiative time $T$ with respect to harmonic time $t$, and enters the relation between the retarded time in radiative coordinates and the retarded time $t-r / c$ in harmonic coordinates (the coordinates in which the equations of motion are given) [48, 49]:

$$
T_{R}=t-\frac{r}{c}-\frac{2 G M}{c^{3}} \ln \left(\frac{r}{r_{0}}\right) .
$$

The remaining radiative multipole moments are given by $38,40,41$. 


$$
\begin{aligned}
\mathcal{U}_{i j k}\left(T_{R}\right)= & \mathcal{M}_{i j k}^{(3)}\left(T_{R}\right)+\frac{2 G M}{c^{3}} \int_{0}^{\infty} d \tau \mathcal{M}_{i j k}^{(5)}\left(T_{R}-\tau\right)\left[\ln \left(\frac{c \tau}{2 r_{0}}\right)+\frac{97}{60}\right]+O\left(\epsilon^{5 / 2}\right), \\
\mathcal{U}_{i j k l}\left(T_{R}\right)= & \mathcal{M}_{i j k l}^{(4)}\left(T_{R}\right)+\frac{2 G M}{c^{3}} \int_{0}^{\infty} d \tau \mathcal{M}_{i j k l}^{(6)}\left(T_{R}-\tau\right)\left[\ln \left(\frac{c \tau}{2 r_{0}}\right)+\frac{59}{30}\right]+\frac{2}{5} \frac{G}{c^{3}} \int_{0}^{\infty} d \tau \mathcal{M}_{\langle i j}^{(3)}\left(T_{R}-\tau\right) \mathcal{M}_{k l\rangle}^{(3)}\left(T_{R}-\tau\right) \\
& -\frac{G}{c^{3}}\left\{\frac{21}{5} \mathcal{M}_{\langle i j}^{(5)} \mathcal{M}_{k l\rangle}+\frac{63}{5} \mathcal{M}_{\langle i j}^{(4)} \mathcal{M}_{k l\rangle}^{(1)}+\frac{102}{5} \mathcal{M}_{\langle i j}^{(3)} \mathcal{M}_{k l\rangle}^{(2)}\right\}+O\left(\epsilon^{5 / 2}\right), \\
\mathcal{U}_{L}= & \mathcal{M}_{L}^{(\ell)}\left(T_{R}\right)+O\left(\epsilon^{3 / 2}\right), \\
\mathcal{V}_{i j}\left(T_{R}\right)= & \mathcal{S}_{i j}^{(2)}\left(T_{R}\right)+\frac{2 G M}{c^{3}} \int_{0}^{\infty} d \tau \mathcal{S}_{i j}^{(4)}\left(T_{R}-\tau\right)\left[\ln \left(\frac{c \tau}{2 r_{0}}\right)+\frac{7}{6}\right]+O\left(\epsilon^{5 / 2}\right), \\
\mathcal{V}_{i j k}\left(T_{R}\right)= & \mathcal{S}_{i j k}^{(3)}\left(T_{R}\right)+\frac{2 G M}{c^{3}} \int_{0}^{\infty} d \tau \mathcal{S}_{i j k}^{(5)}\left(T_{R}-\tau\right)\left[\ln \left(\frac{c \tau}{2 r_{0}}\right)+\frac{5}{3}\right] \\
& +\frac{G}{c^{3}}\left\{\frac{1}{10} \epsilon_{a b\langle i} \mathcal{M}_{j \underline{a}}^{(5)} \mathcal{M}_{k\rangle b}-\frac{1}{2} \epsilon_{a b\langle i} \mathcal{M}_{j \underline{a}}^{(4)} \mathcal{M}_{k\rangle b}^{(1)}-2 \mathcal{S}_{\langle i} \mathcal{M}_{j k\rangle}^{(4)}\right\}+O\left(\epsilon^{5 / 2}\right), \\
\mathcal{V}_{L}= & \mathcal{S}_{L}^{(\ell)}\left(T_{R}\right)+O\left(\epsilon^{3 / 2}\right) .
\end{aligned}
$$

\section{B. Adiabatic inspiral of quasi-circular orbits}

Solving the equations of motion yields explicit expressions for the accelerations of each body in terms of the positions and velocities of the two bodies $[50,51,52,53$, [54, 55, 56, 57, 58, 59, 60]. The two-body equations of motion can then be reduced to relative equations of motion in the center-of-mass frame in terms of the relative position $\vec{x}$ and velocity $\vec{v}[61]$. The relative acceleration $\vec{a}$ is currently known through $3.5 \mathrm{PN}$ order [10, 11]. The effects of radiation reaction (due to the emission of gravitational waves) enters the relative acceleration starting at $2.5 \mathrm{PN}$ order.

The emission of gravitational radiation causes the orbits of an isolated binary system to circularize [62]. Thus for the remainder of this paper the orbital evolution of the binary will be modeled as a slow adiabatic inspiral of a quasi-circular orbit. In addition, it will be assumed that the individual compact objects are non-spinning.

The orbital plane is chosen to be the $X-Y$ plane with the orbital phase $\phi$ defining the direction of the unit vector $\vec{n}=\vec{x} / r$ along the relative separation vector by

$$
\mathbf{n}=\cos \phi \vec{e}_{X}+\sin \phi \vec{e}_{Y}
$$

Then the motion of the binary can be described using the rotating orthonormal triad $\left(\vec{n}, \vec{\lambda}, \vec{e}_{Z}\right)$ with $\vec{\lambda}=\vec{e}_{Z} \times \vec{n}$.

The relative position, velocity, and acceleration are given by

$$
\begin{aligned}
& \vec{x}=r \vec{n}, \\
& \vec{v}=\dot{r} \vec{n}+r \omega \vec{\lambda}, \\
& \vec{a}=\left(\ddot{r}-r \omega^{2}\right) \vec{n}+(r \dot{\omega}+2 \dot{r} \omega) \vec{\lambda},
\end{aligned}
$$

where the orbital frequency $\omega=\dot{\phi}$. Through 2PN order, it is possible to model the motion of the binary as a circular orbit with the solution $\ddot{r}=\dot{r}=\dot{\omega}=0$ and $r \omega^{2}=$ $-\vec{n} \cdot \vec{a}$.

At 2.5PN order, however, the inspiral motion must be taken into account. The leading order contribution to the inspiral of a quasi-circular orbit can be obtained by examining the Newtonian orbital energy of a circular orbit,

$$
E=-\frac{1}{2} \nu \frac{G m^{2}}{r}+O(\epsilon)
$$

where $\nu=m_{1} m_{2} / m^{2}$, and the leading order gravitational luminosity from a circular orbit,

$$
\mathcal{L}=\frac{32}{5} \nu^{2} \frac{G^{4} m^{5}}{r^{5} c^{5}}+O(\epsilon),
$$

and assuming that the energy radiated by the gravitational waves is balanced by the change in the orbital energy (i.e. $d E / d t=-\mathcal{L}$ ). Then

$$
\dot{r}=\left(\frac{d E}{d t} / \frac{d E}{d r}\right)=-\frac{64}{5} \nu \frac{G^{3} m^{3}}{r^{3} c^{5}}+O\left(\epsilon^{7 / 2}\right),
$$

and similarly the orbital frequency changes by

$$
\dot{\omega}=\left(\frac{d \omega}{d r} / \frac{d r}{d t}\right)=\frac{96}{5} \nu\left(\frac{G^{7} m^{7}}{c^{10} r^{11}}\right)^{1 / 2}+O\left(\epsilon^{7 / 2}\right) .
$$

Substituting (37) and (38) into Eqs. (33) and (34) and noting that $\ddot{r}=O\left(\epsilon^{5}\right)$, the following expressions for the $3 \mathrm{PN}$ inspiral relative velocity and relative acceleration are obtained:

$$
\begin{aligned}
& \vec{v}=r \omega \vec{\lambda}-\frac{64}{5} \nu \frac{G^{3} m^{3}}{r^{3} c^{5}} \vec{n}+O\left(\epsilon^{7 / 2}\right), \\
& \vec{a}=-\omega^{2} \vec{x}-\frac{32}{5} \nu \frac{G^{3} m^{3}}{c^{5} r^{4}} \vec{v}+O\left(\epsilon^{7 / 2}\right) .
\end{aligned}
$$


where the $3 \mathrm{PN}$ orbital angular frequency is [54, 55]

$$
\begin{aligned}
\omega^{2}= & \frac{G m}{r^{3}}\left\{1+\gamma(-3+\nu)+\gamma^{2}\left(6+\frac{41}{4} \nu+\nu^{2}\right)\right. \\
& +\gamma^{3}\left(-10+\left[-\frac{75707}{840}+\frac{41}{64} \pi^{2}+22 \ln \left(\frac{r}{r_{0}^{\prime}}\right)\right] \nu\right. \\
& \left.\left.+\frac{19}{2} \nu^{2}+\nu^{3}\right)\right\}+O\left(\epsilon^{4}\right),
\end{aligned}
$$

where the post-Newtonian parameter

$$
\gamma \equiv \frac{G m}{r c^{2}}
$$

and $r_{0}^{\prime}$ is a gauge constant.

As $\gamma$ is defined with respect to the harmonic-coordinate separation $r$, it is convenient to introduce the frequencyrelated post-Newtonian parameter

$$
x \equiv\left(\frac{G m \omega}{c^{3}}\right)^{2 / 3}
$$

Inverting (41) order by order, yields

$$
\begin{aligned}
\gamma= & x\left\{1+x\left(1-\frac{1}{3} \nu\right)+x^{2}\left(1-\frac{65}{12} \nu\right)\right. \\
& +x^{3}\left(1+\left[-\frac{2203}{2520}-\frac{41}{192} \pi^{2}-\frac{22}{3} \ln \left(\frac{r}{r_{0}^{\prime}}\right)\right] \nu\right. \\
& \left.\left.+\frac{229}{36} \nu^{2}+\frac{1}{81} \nu^{3}\right)\right\}+O\left(\epsilon^{4}\right) .
\end{aligned}
$$

\section{Source Multipole Moments}

Defining $\delta m=m_{1}-m_{2}$, the source mass multipole moments for circular orbits are given by [44, 63, 64]

$$
\begin{aligned}
\mathcal{I} & =m\left\{1-\frac{1}{2} \nu \gamma+\gamma^{2} \frac{1}{8} \nu(7-\nu)\right\}+O\left(\epsilon^{3}\right), \\
\mathcal{I}_{i} & =0
\end{aligned}
$$

$$
\begin{aligned}
& \mathcal{I}_{i j}=\nu m\left\{\left[1-\gamma\left(\frac{1}{42}+\frac{13}{14} \nu\right)-\gamma^{2}\left(\frac{461}{1512}+\frac{18395}{1512} \nu+\frac{241}{1512} \nu^{2}\right)\right.\right. \\
& \left.+\gamma^{3}\left(\frac{395899}{13200}-\frac{428}{105} \ln \left(\frac{r}{r_{0}}\right)+\left[\frac{3304319}{166320}-\frac{44}{3} \ln \left(\frac{r}{r_{0}^{\prime}}\right)\right] \nu+\frac{162539}{16632} \nu^{2}+\frac{2351}{33264} \nu^{3}\right)\right] x_{<i j>} \\
& +\left[\frac{11}{21}(1-3 \nu)+\gamma\left(\frac{1607}{378}-\frac{1681}{378} \nu+\frac{229}{378} \nu^{2}\right)+\gamma^{2}\left(\frac{428}{105} \ln \left(\frac{r}{r_{0}}\right)-\frac{357761}{19800}-\frac{92339}{5544} \nu\right.\right. \\
& \left.\left.\left.+\frac{35759}{924} \nu^{2}+\frac{457}{5544} \nu^{3}\right)\right] \frac{r^{2}}{c^{2}} v_{<i j>}+\frac{48}{7} \frac{G^{2} m^{2} \nu}{r c^{5}} x_{<i} v_{j>}\right\}+O\left(\epsilon^{7 / 2}\right), \\
& \mathcal{I}_{i j k}=-\nu \delta m\left\{\left[1-\gamma \nu-\gamma^{2}\left(\frac{139}{330}+\frac{11923}{660} \nu+\frac{29}{110} \nu^{2}\right)\right] x_{<i j k>}\right. \\
& \left.+\left[1-2 \nu+\gamma\left(\frac{1066}{165}-\frac{1433}{330} \nu+\frac{21}{55} \nu^{2}\right)\right] \frac{r^{2}}{c^{2}} x_{<i} v_{j k>}\right\}+O\left(\epsilon^{5 / 2}\right), \\
& \mathcal{I}_{i j k l}=\nu m\left\{\left[1-3 \nu+\gamma\left(\frac{3}{110}-\frac{25}{22} \nu+\frac{69}{22} \nu^{2}\right)-\gamma^{2}\left(\frac{126901}{200200}+\frac{58101}{2600} \nu-\frac{204153}{2860} \nu^{2}-\frac{1149}{1144} \nu^{3}\right)\right] x_{<i j k l>}\right. \\
& +\left[\frac{78}{55}\left(1-5 \nu+5 \nu^{2}\right)+\gamma\left(\frac{30583}{3575}-\frac{107039}{3575} \nu+\frac{8792}{715} \nu^{2}-\frac{639}{715} \nu^{3}\right)\right] \frac{r^{2}}{c^{2}} x_{<i j} v_{k l>} \\
& \left.+\left[\frac{71}{715}\left(1-7 \nu+14 \nu^{2}-7 \nu^{3}\right)\right] \frac{r^{4}}{c^{4}} v_{<i j k l>}\right\}+O\left(\epsilon^{5 / 2}\right), \\
& \mathcal{I}_{i j k l m}=-\nu \delta m\left\{\left[1-2 \nu+\gamma\left(\frac{2}{39}-\frac{47}{39} \nu+\frac{28}{13} \nu^{2}\right)\right] x_{<i j k l m>}+\frac{70}{39}\left(1-4 \nu+3 \nu^{2}\right) \frac{r^{2}}{c^{2}} x_{<i j k} v_{l m>}\right\}+O\left(\epsilon^{2}\right), \\
& \mathcal{I}_{i j k l m n}=\nu m\left\{\left[1-5 \nu+5 \nu^{2}+\gamma\left(\frac{1}{14}-\frac{3}{2} \nu+6 \nu^{2}-\frac{11}{2} \nu^{3}\right)\right] x_{<i j k l m n>}\right. \\
& \left.+\frac{15}{7}\left(1-7 \nu+14 \nu^{2}-7 \nu^{3}\right) \frac{r^{2}}{c^{2}} x_{<i j k l} v_{m n>}\right\}+O\left(\epsilon^{2}\right), \\
& \mathcal{I}_{\text {ijklmno }}=-\nu \delta m\left\{\left[1-4 \nu+3 \nu^{2}+\gamma\left(\frac{3}{34}-\frac{26}{17} \nu+\frac{83}{17} \nu^{2}-\frac{57}{17} \nu^{3}\right)\right] x_{<i j k l m n o>}\right. \\
& \left.+\frac{42}{17}\left(1-6 \nu+10 \nu^{2}-4 \nu^{3}\right) \frac{r^{2}}{c^{2}} x_{<i j k l m} v_{n o>}\right\}+O\left(\epsilon^{2}\right),
\end{aligned}
$$




$$
\begin{aligned}
\mathcal{I}_{\text {ijklmnop }}= & \nu m\left\{\left[1-7 \nu+14 \nu^{2}-7 \nu^{3}+\gamma\left(\frac{35}{342}-\frac{73}{38} \nu+\frac{317}{38} \nu^{2}-\frac{973}{57} \nu^{3}+\frac{301}{38} \nu^{4}\right)\right] x_{<i j k l m n o p>}\right. \\
& \left.\left.+\frac{476}{171}\left(1-9 \nu+27 \nu^{2}-30 \nu^{3}+9 \nu^{4}\right) \frac{r^{2}}{c^{2}} x_{<i j k l m n} v_{o p}\right\rangle\right\}+O\left(\epsilon^{2}\right)
\end{aligned}
$$

through $\ell=8$. In Appendix $\mathrm{A}$ an expression is given for the source mass moments to $1 \mathrm{PN}$ order for arbitrary $\ell$.

The source current multipole moments for circular orbits are [39, 44, 65]

$$
\begin{aligned}
\mathcal{J}_{i}= & L_{i}+O(\epsilon) \\
\mathcal{J}_{i j}= & -\frac{\delta m}{m}\left[1+\gamma\left(\frac{67}{28}-\frac{2}{7} \nu\right)+\gamma^{2}\left(\frac{13}{9}-\frac{4651}{252} \nu-\frac{1}{168} \nu^{2}\right)\right] L_{<i} x_{j>}+O\left(\epsilon^{5 / 2}\right), \\
\mathcal{J}_{i j k}= & {\left[1-3 \nu+\gamma\left(\frac{181}{90}-\frac{109}{18} \nu+\frac{13}{18} \nu^{2}\right)\right] L_{<i} x_{j k>}+\frac{7}{45}\left(1-5 \nu+5 \nu^{2}\right) \frac{r^{2}}{c^{2}} L_{<i} v_{j k>}+O\left(\epsilon^{2}\right), } \\
\mathcal{J}_{i j k l}= & -\frac{\delta m}{m}\left\{\left[1-2 \nu+\gamma\left(\frac{20}{11}-\frac{155}{44} \nu+\frac{5}{11} \nu^{2}\right)\right] L_{<i} x_{j k l>}+\frac{4}{11}\left(1-4 \nu+3 \nu^{2}\right) \frac{r^{2}}{c^{2}} L_{<i} x_{j} v_{k l>}\right\}+O\left(\epsilon^{2}\right)(57) \quad(56) \\
\mathcal{J}_{i j k l m}= & {\left[1-5 \nu+5 \nu^{2}+\gamma\left(\frac{1549}{910}-\frac{1081}{130} \nu+\frac{107}{13} \nu^{2}-\frac{29}{26} \nu^{3}\right)\right] L_{<i} x_{j k l m>} } \\
& +\frac{54}{91}\left(1-7 \nu+14 \nu^{2}-7 \nu^{3}\right) \frac{r^{2}}{c^{2}} L_{<i} x_{j k} v_{l m>}+O\left(\epsilon^{2}\right), \\
\mathcal{J}_{\text {ijklmn }}= & -\frac{\delta m}{m}\left\{1-4 \nu+3 \nu^{2}\right\} L_{<i} x_{j k l m n>}+O(\epsilon), \\
\mathcal{J}_{\text {ijklmno }}= & \left\{1-7 \nu+14 \nu^{2}-7 \nu^{3}\right\} L_{<i} x_{j k l m n o>}+O(\epsilon), \\
\mathcal{J}_{\text {ijklmnop }}= & -\frac{\delta m}{m}\left\{1-6 \nu+10 \nu^{2}-4 \nu^{3}\right\} L_{<i} x_{j k l m n o p>}+O(\epsilon),
\end{aligned}
$$

where $L_{i}=\nu m \epsilon_{i a b} x_{a} v_{b}$. In Appendix B an expression is given for the source current moments to leading order for arbitrary $\ell$.

Finally, the gauge monopolar moment $\mathcal{W}$ for a circular orbit is

$$
\mathcal{W}=\frac{1}{3} \mu x_{a} v_{a}
$$

which is proportional to $\dot{r} \sim O\left(\epsilon^{5 / 2}\right)$. As $\mathcal{W}$ is already a $2.5 \mathrm{PN}$ correction to the source mass quadrupole, it gives no contribution in the present case, and the canonical moments will simply be given by the source moments.

\section{RESULTS}

The computation of the spin-weighted spherical harmonic components is now an exercise in algebra. The evaluation of the tail, memory, and "tail of tail" integrals requires special treatment that is described in Sec. IVA. The spin-weighted spherical harmonic components are listed in Sec. IVB. They will contain terms that depend upon the freely-specifiable constant $r_{0}$ (see the discussion between Eqs. (23) and (24)). These terms can be absorbed into a redefinition of the phase as will be discussed in Sec. IV C.

\section{A. Evaluation of hereditary terms}

The hereditary terms in the radiative multipole moments involve integrals over the entire past history of the binary. These integrals fall into two types, the tail integrals (including the "tail of tail" term) that have logarithmic terms and physically correspond to the backscattering of the gravitational waves off the background curvature [38]; and the memory integrals that can physically be thought of as the re-radiation of the stress-energy of the propagating waves [45, 46, 47].

Inserting the canonical multipole moments into the tail integrals of the radiative multipole moments yields expressions of the form

$$
I_{1}\left(T_{R}\right)=\int_{0}^{\infty} d \tau F\left(T_{R}-\tau\right) e^{-i k \omega\left(T_{R}-\tau\right)}\left[\ln \left(\frac{c \tau}{2 r_{0}}\right)+b\right]
$$

where $k$ is an integer (the index $m$ of the $h^{\ell m}$ being computed $), b$ is a rational number, and $F\left(T_{R}-\tau\right)$ represents a function whose time dependence enters only through its dependence on the orbital frequency. In [12, 49], it has been shown that the oscillatory term in the integrand combined with the slow adiabatic evolution of the orbital frequency allow the integral to be approximated by using the orbital frequency of a fixed circular orbit at 
the current value of $T_{R}$, so that

$$
\begin{aligned}
I_{1}\left(T_{R}\right)= & F\left(T_{R}\right) e^{-i k \phi}\left\{\int_{0}^{\infty} d \tau e^{i k \omega \tau}\left[\ln \left(\frac{c \tau}{2 r_{0}}\right)+b\right]\right. \\
& +O(\xi \ln \xi)\},
\end{aligned}
$$

where $\xi \sim \dot{\omega} / \omega^{2}$, is the adiabatic parameter describing the decay of the orbit which is $O\left(\epsilon^{5 / 2}\right)$. Equation (64) can be evaluated using the identity

$$
\int_{0}^{\infty} d \tau \ln \tau e^{-\sigma \tau}=-\frac{1}{\sigma}\left(\gamma_{E}+\ln \sigma\right)
$$

where $\gamma_{E}$ is Euler's constant. Using (65) to evaluate (64) yields

$$
\begin{aligned}
I_{1}\left(T_{R}\right)= & -\frac{1}{k \omega} F\left(T_{R}\right) e^{-i k \phi}\left\{\frac{\pi}{2}\right. \\
& \left.+i\left[\gamma_{E}+\ln \left(\frac{2 k \omega r_{0}}{c}\right)-b\right]\right\} .
\end{aligned}
$$

For reasons that will be explained in Sec. IV C, it is convenient to express the above result as

$$
\begin{aligned}
I_{1}\left(T_{R}\right)= & -\frac{1}{k \omega} F\left(T_{R}\right) e^{-i k \phi}\left\{\frac{\pi}{2}\right. \\
& \left.+i\left[\frac{3}{2} \ln \left(\frac{x}{x_{0}}\right)+\ln \frac{k}{2}+\frac{11}{12}-b\right]\right\},
\end{aligned}
$$

where

$$
\ln x_{0} \equiv \frac{11}{18}-\frac{2}{3} \gamma_{E}-\frac{4}{3} \ln 2+\frac{2}{3} \ln \left(\frac{G m}{c^{2} r_{0}}\right) .
$$

A similar argument holds for the "tail of tail" integral in Eq. (23) where a term of the form

$$
I_{2}\left(T_{R}\right)=\int_{0}^{\infty} d \tau F\left(T_{R}-\tau\right) e^{-i k \omega\left(T_{R}-\tau\right)}\left[\ln \left(\frac{c \tau}{2 r_{0}}\right)\right]^{2}
$$

is found. In this case the integral can be evaluated with the aid of the identity

$$
\int_{0}^{\infty} d \tau(\ln \tau)^{2} e^{-\sigma \tau}=\frac{1}{\sigma}\left[\frac{\pi^{2}}{6}+\left(\gamma_{E}+\ln \sigma\right)^{2}\right],
$$

to yield

$$
\begin{aligned}
I_{2}\left(T_{R}\right)= & \frac{1}{k \omega} F\left(T_{R}\right) e^{-i k \phi}\left\{\pi\left[\frac{3}{2} \ln \left(\frac{x}{x_{0}}\right)+\ln \frac{k}{2}+\frac{11}{12}\right]\right. \\
& \left.+i\left[\frac{3}{2} \ln \left(\frac{x}{x_{0}}\right)+\ln \frac{k}{2}+\frac{11}{12}\right]^{2}\right\} .
\end{aligned}
$$

The memory integral in Eq. (26) leads to an integral of the form

$$
I_{3}\left(T_{R}\right)=\int_{0}^{\infty} F\left(T_{R}-\tau\right) e^{-i k \omega\left(T_{R}-\tau\right)} d \tau .
$$

Using similar arguments as for the tail integrals, Ref. [12] has shown that this integral can be approximated by

$$
I_{3}\left(T_{R}\right)=\frac{i}{k \omega} F\left(T_{R}\right) e^{-i k \phi}[1+O(\xi)] .
$$

The memory integrals also lead to an integral of the form

$$
I_{4}\left(T_{R}\right)=\int_{0}^{\infty} x^{5}\left(T_{R}-\tau\right) d \tau
$$

Unlike the other integrals, it does not depend upon the orbital phase, and thus is much more sensitive to the past history of the binary. This is the non-linear memory effect described in 45, 46, 47]. This integral has been evaluated by [12, 46] using the evolution of the frequency parameter $x$ found by integrating Eq. 38

$$
x(t)=\frac{1}{4}\left[\left(\frac{\nu c^{3}}{5 G m}\right)\left(t_{c}-t\right)\right]^{-1 / 4}[1+O(\epsilon)]
$$

where $t_{c}$ denotes the time of coalescence. With this model,

$$
I_{4}\left(T_{R}\right)=\frac{5}{64} \frac{G m}{\nu c^{3}} x[1+O(\epsilon)]
$$

As discussed in 12, 38, 46], this contribution to the waveform will be very difficult to detect as it is essentially DC in character corresponding to a steadily-growing part of the waveform with no dependence on the orbital phase. It does, however, build up during the entire inspiral which leads to its magnitude being comparable with the $0 \mathrm{PN}$ quadrupolar term despite arising formally as a $2.5 \mathrm{PN}$ contribution. See [47] for a discussion on strategies for detecting the memory piece of the waveform.

\section{B. Spin-weighted spherical harmonic components}

Examining Eq. (19), seems to show that the individual spin-weighted spherical harmonic components could obtain contributions from both mass and current radiative multipoles. It turns out for the non-spinning case, however, that the mass (current) multipoles only contribute to components with $\ell+m$ even (odd). Because of this separation, and since [24] $U^{\ell m *}=(-1)^{m} U^{\ell,-m}$ and $V^{\ell m *}=(-1)^{m} V^{\ell,-m}$, it follows from Eq. (19) that

$$
h^{\ell,-m}= \begin{cases}(-1)^{m} h^{\ell m *} & (\ell+m \text { even }) \\ (-1)^{m+1} h^{\ell m *} & (\ell+m \text { odd })\end{cases}
$$

which simply reduces to

$$
h^{\ell,-m}=(-1)^{\ell} h^{\ell m *}
$$

Substituting the source mass quadrupole (47) into the expression for the radiative mass quadrupole (23), taking the appropriate time derivatives, substituting the equations of motion (40), and evaluating the hereditary integrals using the techniques described in Sec. IVA, the dominant component of the waveform is given to $3 \mathrm{PN}$ order as 


$$
\begin{aligned}
h^{22}= & -8 \sqrt{\frac{\pi}{5}} \frac{G \nu m}{c^{2} R} e^{-2 i \phi} x\left\{1-x\left(\frac{107}{42}-\frac{55}{42} \nu\right)+x^{3 / 2}\left[2 \pi+6 i \ln \left(\frac{x}{x_{0}}\right)\right]-x^{2}\left(\frac{2173}{1512}+\frac{1069}{216} \nu-\frac{2047}{1512} \nu^{2}\right)\right. \\
& -x^{5 / 2}\left[\left(\frac{107}{21}-\frac{34}{21} \nu\right) \pi+24 i \nu+\left(\frac{107 i}{7}-\frac{34 i}{7} \nu\right) \ln \left(\frac{x}{x_{0}}\right)\right]+x^{3}\left[\frac{27027409}{646800}-\frac{856}{105} \gamma_{E}+\frac{2}{3} \pi^{2}-\frac{1712}{105} \ln 2\right. \\
& \left.-\frac{428}{105} \ln x-18\left[\ln \left(\frac{x}{x_{0}}\right)\right]^{2}-\left(\frac{278185}{33264}-\frac{41}{96} \pi^{2}\right) \nu-\frac{20261}{2772} \nu^{2}+\frac{114635}{99792} \nu^{3}+\frac{428 i}{105} \pi+12 i \pi \ln \left(\frac{x}{x_{0}}\right)\right] \\
& \left.+O\left(\epsilon^{7 / 2}\right)\right\}
\end{aligned}
$$

where the constant $r_{0}$ has been eliminating from the expression by using Eq. (68). The remaining components are given by

$$
\begin{aligned}
& h^{21}=-\frac{8 i}{3} \sqrt{\frac{\pi}{5}} \frac{G \nu \delta m}{c^{2} R} e^{-i \phi} x^{3 / 2}\left\{1-x\left(\frac{17}{28}-\frac{5}{7} \nu\right)+x^{3 / 2}\left[\pi-\frac{i}{2}-2 i \ln 2+3 i \ln \left(\frac{x}{x_{0}}\right)\right]\right. \\
& \left.-x^{2}\left(\frac{43}{126}+\frac{509}{126} \nu-\frac{79}{168} \nu^{2}\right)+O\left(\epsilon^{5 / 2}\right)\right\} \text {, } \\
& h^{20}=\frac{2}{7} \sqrt{\frac{10 \pi}{3}} \frac{G \nu m}{c^{2} R} x[1+O(\epsilon)] \text {, } \\
& h^{33}=3 i \sqrt{\frac{6 \pi}{7}} \frac{G \nu \delta m}{c^{2} R} e^{-3 i \phi} x^{3 / 2}\left\{1-x(4-2 \nu)+x^{3 / 2}\left[3 \pi-\frac{21 i}{5}+6 i \ln \frac{3}{2}+9 i \ln \left(\frac{x}{x_{0}}\right)\right]\right. \\
& \left.+x^{2}\left(\frac{123}{110}-\frac{1838}{165} \nu+\frac{887}{330} \nu^{2}\right)+O\left(\epsilon^{5 / 2}\right)\right\}, \\
& h^{32}=-\frac{8}{3} \sqrt{\frac{\pi}{7}} \frac{G \nu m}{c^{2} R} e^{-2 i \phi} x^{2}\left\{1-3 \nu-x\left(\frac{193}{90}-\frac{145}{18} \nu+\frac{73}{18} \nu^{2}\right)\right. \\
& \left.+x^{3 / 2}\left[2 \pi(1-3 \nu)-3 i+\frac{66 i}{5} \nu+6 i(1-3 \nu) \ln \left(\frac{x}{x_{0}}\right)\right]+O\left(\epsilon^{2}\right)\right\} \text {, } \\
& h^{31}=-\frac{i}{3} \sqrt{\frac{2 \pi}{35}} \frac{G \nu \delta m}{c^{2} R} e^{-i \phi} x^{3 / 2}\left\{1-x\left(\frac{8}{3}+\frac{2}{3} \nu\right)+x^{3 / 2}\left[\pi-\frac{7 i}{5}-2 i \ln 2+3 i \ln \left(\frac{x}{x_{0}}\right)\right]\right. \\
& \left.+x^{2}\left(\frac{607}{198}-\frac{136}{99} \nu-\frac{247}{198} \nu^{2}\right)+O\left(\epsilon^{5 / 2}\right)\right\}, \\
& h^{30}=\frac{16 i}{5} \sqrt{\frac{6 \pi}{35}} \frac{G \nu m}{c^{2} R} \nu x^{7 / 2}[1+O(\epsilon)] \text {, } \\
& h^{44}=\frac{64}{9} \sqrt{\frac{\pi}{7}} \frac{G \nu m}{c^{2} R} e^{-4 i \phi} x^{2}\left\{1-3 \nu-x\left(\frac{593}{110}-\frac{1273}{66} \nu+\frac{175}{22} \nu^{2}\right)\right. \\
& +x^{3 / 2}\left[4 \pi(1-3 \nu)-\frac{42 i}{5}+\frac{1193 i}{40} \nu+8 i(1-3 \nu) \ln 2+12 i(1-3 \nu) \ln \left(\frac{x}{x_{0}}\right)\right] \\
& \left.+x^{2}\left(\frac{1068671}{200200}-\frac{1088119}{28600} \nu+\frac{146879}{2340} \nu^{2}-\frac{226097}{17160} \nu^{3}\right)+O\left(\epsilon^{5 / 2}\right)\right\}, \\
& h^{43}=\frac{9 i}{5} \sqrt{\frac{2 \pi}{7}} \frac{G \nu \delta m}{c^{2} R} e^{-3 i \phi} x^{5 / 2}\left\{1-2 \nu-x\left(\frac{39}{11}-\frac{1267}{132} \nu+\frac{131}{33} \nu^{2}\right)+O\left(\epsilon^{3 / 2}\right)\right\}, \\
& h^{42}=-\frac{8}{63} \sqrt{\pi} \frac{G \nu m}{c^{2} R} e^{-2 i \phi} x^{2}\left\{1-3 \nu-x\left(\frac{437}{110}-\frac{805}{66} \nu+\frac{19}{22} \nu^{2}\right)\right. \\
& +x^{3 / 2}\left[2 \pi(1-3 \nu)-\frac{21 i}{5}+\frac{84 i}{5} \nu+6 i(1-3 \nu) \ln \left(\frac{x}{x_{0}}\right)\right] \\
& \left.+x^{2}\left(\frac{1038039}{200200}-\frac{606751}{28600} \nu+\frac{400453}{25740} \nu^{2}+\frac{25783}{17160} \nu^{3}\right)+O\left(\epsilon^{5 / 2}\right)\right\}, \\
& h^{41}=-\frac{i}{105} \sqrt{2 \pi} \frac{G \nu \delta m}{c^{2} R} e^{-i \phi} x^{5 / 2}\left\{1-2 \nu-x\left(\frac{101}{33}-\frac{337}{44} \nu+\frac{83}{33} \nu^{2}\right)+O\left(\epsilon^{3 / 2}\right)\right\},
\end{aligned}
$$




$$
\begin{aligned}
& h^{40}=\frac{1}{63} \sqrt{\frac{\pi}{10}} \frac{G \nu m}{c^{2} R} x[1+O(\epsilon)], \\
& h^{55}=-\frac{125 i}{12} \sqrt{\frac{5 \pi}{66}} \frac{G \nu \delta m}{c^{2} R} e^{-5 i \phi} x^{5 / 2}\left\{1-2 \nu-x\left(\frac{263}{39}-\frac{688}{39} \nu+\frac{256}{39} \nu^{2}\right)+O\left(\epsilon^{3 / 2}\right)\right\}, \\
& h^{54}=\frac{256}{45} \sqrt{\frac{\pi}{33}} \frac{G \nu m}{c^{2} R} e^{-4 i \phi} x^{3}\left\{1-5 \nu+5 \nu^{2}-x\left(\frac{4451}{910}-\frac{3619}{130} \nu+\frac{521}{13} \nu^{2}-\frac{339}{26} \nu^{3}\right)+O\left(\epsilon^{3 / 2}\right)\right\}, \\
& h^{53}=\frac{9 i}{20} \sqrt{\frac{3 \pi}{22}} \frac{G \nu \delta m}{c^{2} R} e^{-3 i \phi} x^{5 / 2}\left\{1-2 \nu-x\left(\frac{69}{13}-\frac{464}{39} \nu+\frac{88}{39} \nu^{2}\right)+O\left(\epsilon^{3 / 2}\right)\right\} \text {, } \\
& h^{52}=-\frac{16}{135} \sqrt{\frac{\pi}{11}} \frac{G \nu m}{c^{2} R} e^{-2 i \phi} x^{3}\left\{1-5 \nu+5 \nu^{2}-x\left(\frac{3911}{910}-\frac{3079}{130} \nu+\frac{413}{13} \nu^{2}-\frac{231}{26} \nu^{3}\right)+O\left(\epsilon^{3 / 2}\right)\right\}, \\
& h^{51}=-\frac{i}{180} \sqrt{\frac{\pi}{77}} \frac{G \nu \delta m}{c^{2} R} e^{-i \phi} x^{5 / 2}\left\{1-2 \nu-x\left(\frac{179}{39}-\frac{352}{39} \nu+\frac{4}{39} \nu^{2}\right)+O\left(\epsilon^{3 / 2}\right)\right\}, \\
& h^{66}=-\frac{432}{5} \sqrt{\frac{\pi}{715}} \frac{G \nu m}{c^{2} R} e^{-6 i \phi} x^{3}\left\{1-5 \nu+5 \nu^{2}-x\left(\frac{113}{14}-\frac{91}{2} \nu+64 \nu^{2}-\frac{39}{2} \nu^{3}\right)+O\left(\epsilon^{3 / 2}\right)\right\}, \\
& h^{65}=-\frac{625 i}{63} \sqrt{\frac{5 \pi}{429}} \frac{G \nu \delta m}{c^{2} R} e^{-5 i \phi} x^{7 / 2}\left\{1-4 \nu+3 \nu^{2}+O(\epsilon)\right\}, \\
& h^{64}=\frac{1024}{495} \sqrt{\frac{2 \pi}{195}} \frac{G \nu m}{c^{2} R} e^{-4 i \phi} x^{3}\left\{1-5 \nu+5 \nu^{2}-x\left(\frac{93}{14}-\frac{71}{2} \nu+44 \nu^{2}-\frac{19}{2} \nu^{3}\right)+O\left(\epsilon^{3 / 2}\right)\right\}, \\
& h^{63}=\frac{81 i}{385} \sqrt{\frac{\pi}{13}} \frac{G \nu \delta m}{c^{2} R} e^{-3 i \phi} x^{7 / 2}\left\{1-4 \nu+3 \nu^{2}+O(\epsilon)\right\}, \\
& h^{62}=-\frac{16}{1485} \sqrt{\frac{\pi}{13}} \frac{G \nu m}{c^{2} R} e^{-2 i \phi} x^{3}\left\{1-5 \nu+5 \nu^{2}-x\left(\frac{81}{14}-\frac{59}{2} \nu+32 \nu^{2}-\frac{7}{2} \nu^{3}\right)+O\left(\epsilon^{3 / 2}\right)\right\}, \\
& h^{61}=-\frac{i}{2079} \sqrt{\frac{2 \pi}{65}} \frac{G \nu \delta m}{c^{2} R} e^{-i \phi} x^{7 / 2}\left\{1-4 \nu+3 \nu^{2}+O(\epsilon)\right\} \\
& h^{77}=\frac{16807 i}{180} \sqrt{\frac{7 \pi}{4290}} \frac{G \nu \delta m}{c^{2} R} e^{-7 i \phi} x^{7 / 2}\left\{1-4 \nu+3 \nu^{2}-x\left(\frac{319}{34}-\frac{2225}{51} \nu+\frac{2558}{51} \nu^{2}-\frac{230}{17} \nu^{3}\right)+O\left(\epsilon^{3 / 2}\right)\right\}, \\
& h^{76}=-\frac{648}{35} \sqrt{\frac{3 \pi}{715}} \frac{G \nu m}{c^{2} R} e^{-6 i \phi} x^{4}\left\{1-7 \nu+14 \nu^{2}-7 \nu^{3}+O(\epsilon)\right\} \text {, } \\
& h^{75}=-\frac{3125 i}{3276} \sqrt{\frac{5 \pi}{66}} \frac{G \nu \delta m}{c^{2} R} e^{-5 i \phi} x^{7 / 2}\left\{1-4 \nu+3 \nu^{2}-x\left(\frac{271}{34}-\frac{1793}{51} \nu+\frac{1838}{51} \nu^{2}-\frac{134}{17} \nu^{3}\right)+O\left(\epsilon^{3 / 2}\right)\right\}, \\
& h^{74}=\frac{1024}{1365} \sqrt{\frac{2 \pi}{165}} \frac{G \nu m}{c^{2} R} e^{-4 i \phi} x^{4}\left\{1-7 \nu+14 \nu^{2}-7 \nu^{3}+O(\epsilon)\right\} \\
& h^{73}=\frac{243 i}{20020} \sqrt{\frac{3 \pi}{10}} \frac{G \nu \delta m}{c^{2} R} e^{-3 i \phi} x^{7 / 2}\left\{1-4 \nu+3 \nu^{2}-x\left(\frac{239}{34}-\frac{1505}{51} \nu+\frac{1358}{51} \nu^{2}-\frac{70}{17} \nu^{3}\right)+O\left(\epsilon^{3 / 2}\right)\right\}, \\
& h^{72}=-\frac{8}{3003} \sqrt{\frac{\pi}{15}} \frac{G \nu m}{c^{2} R} e^{-2 i \phi} x^{4}\left\{1-7 \nu+14 \nu^{2}-7 \nu^{3}+O(\epsilon)\right\}, \\
& h^{71}=-\frac{i}{108108} \sqrt{\frac{\pi}{10}} \frac{G \nu \delta m}{c^{2} R} e^{-i \phi} x^{7 / 2}\left\{1-4 \nu+3 \nu^{2}-x\left(\frac{223}{34}-\frac{1361}{51} \nu+\frac{1118}{51} \nu^{2}-\frac{38}{17} \nu^{3}\right)+O\left(\epsilon^{3 / 2}\right)\right\}, \\
& h^{88}=\frac{131072}{315} \sqrt{\frac{2 \pi}{17017}} \frac{G \nu m}{c^{2} R} e^{-8 i \phi} x^{4}\left\{1-7 \nu+14 \nu^{2}-7 \nu^{3}-x\left(\frac{3653}{342}-\frac{9325}{114} \nu+\frac{22351}{114} \nu^{2}-\frac{9107}{57} \nu^{3}+\frac{4081}{114} \nu^{4}\right)\right. \\
& \left.+O\left(\epsilon^{3 / 2}\right)\right\} \text {, } \\
& h^{87}=\frac{117649 i}{3240} \sqrt{\frac{7 \pi}{4862}} \frac{G \nu \delta m}{c^{2} R} e^{-7 i \phi} x^{9 / 2}\left\{1-6 \nu+10 \nu^{2}-4 \nu^{3}+O(\epsilon)\right\} \\
& h^{86}=-\frac{1944}{35} \sqrt{\frac{3 \pi}{85085}} \frac{G \nu m}{c^{2} R} e^{-6 i \phi} x^{4}\left\{1-7 \nu+14 \nu^{2}-7 \nu^{3}-x\left(\frac{353}{38}-\frac{7897}{114} \nu+\frac{18067}{114} \nu^{2}-\frac{6727}{57} \nu^{3}+\frac{2653}{114} \nu^{4}\right)\right. \\
& \left.+O\left(\epsilon^{3 / 2}\right)\right\}
\end{aligned}
$$




$$
\begin{aligned}
& h^{85}=-\frac{15625 i}{4536} \sqrt{\frac{5 \pi}{4862}} \frac{G \nu \delta m}{c^{2} R} e^{-5 i \phi} x^{9 / 2}\left\{1-6 \nu+10 \nu^{2}-4 \nu^{3}+O(\epsilon)\right\}, \\
& h^{84}=\frac{1024}{4095} \sqrt{\frac{2 \pi}{935}} \frac{G \nu m}{c^{2} R} e^{-4 i \phi} x^{4}\left\{1-7 \nu+14 \nu^{2}-7 \nu^{3}-x\left(\frac{2837}{342}-\frac{6877}{114} \nu+\frac{15007}{114} \nu^{2}-\frac{5027}{57} \nu^{3}+\frac{1633}{114} \nu^{4}\right)\right. \\
& \left.+O\left(\epsilon^{3 / 2}\right)\right\} \\
& h^{83}=\frac{81 i}{3640} \sqrt{\frac{3 \pi}{374}} \frac{G \nu \delta m}{c^{2} R} e^{-3 i \phi} x^{9 / 2}\left\{1-6 \nu+10 \nu^{2}-4 \nu^{3}+O(\epsilon)\right\}, \\
& h^{82}=-\frac{8}{45045} \sqrt{\frac{\pi}{17}} \frac{G \nu m}{c^{2} R} e^{-2 i \phi} x^{4}\left\{1-7 \nu+14 \nu^{2}-7 \nu^{3}-x\left(\frac{2633}{342}-\frac{6265}{114} \nu+\frac{13171}{114} \nu^{2}-\frac{4007}{57} \nu^{3}+\frac{1021}{114} \nu^{4}\right)\right. \\
& \left.+O\left(\epsilon^{3 / 2}\right)\right\} \text {, } \\
& h^{81}=-\frac{i}{92664} \sqrt{\frac{\pi}{1190}} \frac{G \nu \delta m}{c^{2} R} e^{-i \phi} x^{9 / 2}\left\{1-6 \nu+10 \nu^{2}-4 \nu^{3}+O(\epsilon)\right\} \text {. }
\end{aligned}
$$

In Appendix C] spin-weighted harmonic components for even (odd) $\ell+m$ are given to $1 \mathrm{PN}(0 \mathrm{PN})$ order for arbitrary $\ell$. Again, the $m<0$ components are given by (78).

\section{Absorbing amplitude terms into the phase}

The $\ln \left(x / x_{0}\right)$ terms that appear in the spin-weighted spherical harmonic components can be absorbed into a redefinition of the phase by introducing an auxiliary phase variable $\psi=\phi+\delta$. Since the $\ln \left(x / x_{0}\right)$ terms first enter at $1.5 \mathrm{PN}$ order, it is straightforward to show that choosing [12, 43]

$$
\delta=-3 \frac{M}{m} x^{3 / 2} \ln \left(\frac{x}{x_{0}}\right),
$$

where $M=\mathcal{I}$ (the mass monopole of the source), will eliminate the $\ln \left(x / x_{0}\right)$ terms from the components. This follows from

$$
\begin{aligned}
h^{\ell m} & =\tilde{h}^{\ell m} e^{-i m \psi} \\
& =\tilde{h}^{\ell m} e^{-i m \phi} e^{-i m \delta} \\
& =\tilde{h}^{\ell m} e^{-i m \phi}\left[1-i m \delta-\frac{1}{2} m^{2} \delta^{2}+O\left(x^{9 / 2}\right)\right]
\end{aligned}
$$

where $\tilde{h}^{\ell m}$ is $h^{\ell m}$ omitting the $\ln \left(x / x_{0}\right)$ terms. Furthermore, since the orbital phase as a function of frequency goes as

$$
\phi=-\frac{1}{32 \nu} x^{-5 / 2}+O(\epsilon)
$$

at leading order, the $\ln \left(x / x_{0}\right)$ terms, which were $1.5 \mathrm{PN}$, $2.5 \mathrm{PN}$, and $3 \mathrm{PN}$ order in the amplitude terms, now appear as phase corrections at relative order $4 \mathrm{PN}, 5 \mathrm{PN}$, and 5.5PN. As these terms are beyond the order to which the orbital phase evolution is known (3.5PN order), it can be argued that these terms can be ignored. Note that the choices of $x_{0}$ in Eq. (68) and $\delta$ in Eq. (117) are not unique as other amplitude terms can be absorbed into the phase (e.g. see [13]); these choices were made to gather all logarithmic terms into one term, as well as to simplify the waveform 43].

In order to recover the $2.5 \mathrm{PN}$ polarization waveforms in [12, 13] from the components listed above, it is necessary to substitute $\ln \left(x / x_{0}\right) \rightarrow 0$. After substituting the coefficients into Eq. (11) and truncating the sum at $2.5 \mathrm{PN}$ order, the result must be evaluated at $\Theta=i$, $\Phi=\pi / 2$. Furthermore, there is an overall sign difference due to a different choice of the polarization triad $(\vec{N}, \vec{P}, \vec{Q})$.

The polarization waveforms have been computed in the limit $\nu \rightarrow 0$ in [7] using black hole perturbation theory. In order to compare with their results, it is necessary to substitute

$$
\ln \left(\frac{x}{x_{0}}\right) \rightarrow-\frac{17}{18}+\frac{2}{3} \ln 2,
$$

into the $h^{\ell m}$ listed above as [7] makes a different choice in redefining the phase variable (and works in Schwarzschild coordinates as opposed to harmonic coordinates). After this substitution, and setting $\nu=0$ and $\delta m / m=-1$, it is found that the $h^{\ell m}$ above agree with the results of [7, 66]. ${ }^{2}$

\section{CONCLUSIONS}

It has been shown that the spin-weighted spherical harmonic components of the waveform can be computed to higher post-Newtonian order by computing them directly from the radiative multipole moments rather than by projecting them from the full polarization waveforms. In

\footnotetext{
2 There is a sign difference in $\zeta_{+}^{\ell, m}$ between [66] and [7]. The results presented here agree with the sign of [66].
} 
particular, this allows the dominant $h^{22}$ component to be computed to $3 \mathrm{PN}$ order. Since numerical simulations can compute the waveform over the entire sphere, it is possible to compare the spin-weighted spherical harmonic components of the waveform from the simulation with those predicted by a quasi-adiabatic post-Newtonian inspiral. Thus, by examining the $h^{22}$ component, it can be determined whether or not the $3 \mathrm{PN}$ contribution improves the agreement between the post-Newtonian waveform and the numerical waveform. If significant improvement is found, it would suggest that it would be worth the effort of computing the full $3 \mathrm{PN}$ waveform in order to improve detection of marginal signals [25, 26, 27] as well as improve parameter extraction [28, 29, 30, 31, 32, 33, 34].

In [23] a high-accuracy comparison is made between post-Newtonian generated waveforms and waveforms from a numerical simulation of 15 orbits of an inspiral of an equal-mass non-spinning binary black hole system. For this case, 23] finds that the $3 \mathrm{PN}$ contributions to the amplitude of the $h^{22}$ mode improve the accuracy with respect to the numerical waveforms. This suggests that for accurate parameter estimation, it may be desirable to compute the full $3 \mathrm{PN}$ amplitude for the polarization waveforms.

For an equal-mass, non-spinning binary, only the source current octupole needs to be computed to have the full polarization waveform. For a non-spinning binary with an arbitrary mass ratio, much more effort is required as explained at the end of [12]. But for comparison with numerical simulations, post-Newtonian theorists should keep in mind that extending the PN order of a given radiative multipole moment, will result in corresponding improvements in the spin-weighted spherical harmonic components of the waveform, and these corrections will be of interest even if all the corrections needed to improve the polarization waveforms to the next order have yet to be computed.

\section{Acknowledgments}

I would like to thank Michael Boyle, Gregory Cook, Abdul Mroué and Saul Teukolsky for helpful discussions concerning this work. This work was supported in part by a grant from the Sherman Fairchild Foundation, by NSF grants PHY-0652952, DMS-0553677, PHY-0652929, and NASA grant NNG05GG51G.

\section{APPENDIX A: 1PN SOURCE MASS MOMENTS}

The source mass multipole moments for a system of $N$ (nonrotating) compact point-masses to $1 \mathrm{PN}$ order is given by [44, 63, 64]

$$
\begin{aligned}
\mathcal{I}_{L}= & \sum_{A=1}^{N}\left\{\tilde{\mu}_{A} y_{A}^{<L>}+\frac{1}{2(2 \ell+3) c^{2}} \frac{d^{2}}{d t^{2}}\left[m_{A} y_{A}^{2} y_{A}^{<L>}\right]\right. \\
& \left.-\frac{4(2 \ell+1)}{(\ell+1)(2 \ell+3) c^{2}} \frac{d}{d t}\left[m_{A} v_{A}^{a} y_{A}^{<a L>}\right]+O\left(\epsilon^{2}\right)\right\},
\end{aligned}
$$

where $m_{A}, \vec{y}_{A}$, and $\vec{v}_{A}$ are the mass, position, and velocity respectively of the $A$-th point mass, and

$$
\tilde{\mu}_{A}=m_{A}\left[1+\frac{3}{2} \frac{v_{A}^{2}}{c^{2}}-\frac{G}{c^{2}} \sum_{B \neq A} \frac{m_{B}}{\left|\vec{y}_{A}-\vec{y}_{B}\right|}+O\left(\epsilon^{2}\right)\right] .
$$

Restricting to the case of two bodies in a quasi-circular orbit, and transforming to the center-of-mass frame using

$$
\begin{aligned}
& \vec{y}_{1}=\frac{m_{2}}{m} \vec{x}\left[1+O\left(\epsilon^{2}\right)\right], \\
& \vec{y}_{2}=-\frac{m_{1}}{m} \vec{x}\left[1+O\left(\epsilon^{2}\right)\right],
\end{aligned}
$$

and using the relative equation of motion (34) to eliminate the relative acceleration from the derivatives yields

$$
\mathcal{I}_{L}=\nu \tilde{m}\left\{\left[f_{\ell-1}(\nu)-\gamma f_{\ell}(\nu)+\gamma \frac{5 \ell^{2}+6 \ell+9}{2(\ell+1)(2 \ell+3)} f_{\ell+1}(\nu)\right] x_{<L>}+\frac{\ell(\ell-1)(\ell+9)}{2(\ell+1)(2 \ell+3)} f_{\ell+1}(\nu) \frac{r^{2}}{c^{2}} x_{<L-2} v_{i_{\ell-1} i_{\ell}>}+O\left(\epsilon^{2}\right)\right\}
$$

where

$$
\left\{\tilde{m}, f_{k}(\nu)\right\}= \begin{cases}\left\{m, s_{k}(\nu)\right\} & \text { for } \ell \text { even } \\ \left\{-\delta m, d_{k}(\nu)\right\} & \text { for } \ell \text { odd }\end{cases}
$$

where $s_{\ell}=\left(m_{1}^{\ell}+m_{2}^{\ell}\right) / m^{\ell}$ and $d_{\ell}=\left(m_{1}^{\ell}-m_{2}^{\ell}\right) / m^{\ell}$, which themselves can be rewritten as polynomials in $\nu$ as

$$
\begin{aligned}
& s_{\ell}(\nu)=1+\sum_{k=1}^{\ell / 2}\left[\left(\begin{array}{c}
\ell-k-1 \\
k-1
\end{array}\right)+\left(\begin{array}{c}
\ell-k \\
k
\end{array}\right)\right](-\nu)^{k} \\
& d_{\ell}(\nu)=\sum_{k=0}^{\ell / 2}\left(\begin{array}{c}
\ell-k-1 \\
k
\end{array}\right)(-\nu)^{k} .
\end{aligned}
$$




\section{APPENDIX B: OPN SOURCE CURRENT MOMENTS}

The source current multipole moments for a system of $\mathrm{N}$ (nonspinning) compact point-masses is given to $0 \mathrm{PN}$ order by 44,65

$$
\mathcal{J}_{L}=\sum_{A=1}^{N} m_{A} \epsilon^{a b<i_{\ell}} y_{A}^{L-1>a} v_{A}^{b}+O(\epsilon) .
$$

Restricting to the case of two bodies in a quasi-circular orbit, and transforming to the center-of-mass frame as for the mass moments in Appendix A, it is straightforward to show that the source current multipole moments to leading order are given by

$$
\mathcal{J}_{L}=\frac{\hat{m}}{m} g_{\ell}(\nu) L_{<i_{\ell}} x_{L-1>}+O(\epsilon),
$$

where

$$
\left\{\hat{m}, g_{k}(\nu)\right\}= \begin{cases}\left\{-\delta m, d_{k}(\nu)\right\} & \text { for } \ell \text { even } \\ \left\{m, s_{k}(\nu)\right\} & \text { for } \ell \text { odd }\end{cases}
$$

\section{APPENDIX C: 1PN SPIN-WEIGHTED SPHERICAL HARMONIC COMPONENTS}

\section{1. $\ell+m$ even}

For even $\ell+m$ (for non-spinning binaries), the spinweighted spherical harmonic components are due to con- tributions from the radiative mass multipoles. To $1 \mathrm{PN}$ order, the radiative moments are given simply $\ell$ time derivatives of the source moments. For arbitrary $\ell$, the mass moment is given by Eq. (A5). In evaluating the spin-weighted spherical harmonic components from the source mass moments, the following identity is useful:

$$
\begin{aligned}
x_{L} \mathcal{Y}_{L}^{\ell m} & =r^{\ell} n_{L} \mathcal{Y}_{L}^{\ell m} \\
& =r^{\ell} Y^{\ell m}\left(\frac{\pi}{2}, \phi\right),
\end{aligned}
$$

where $\phi$ is the orbital phase (as opposed to an angle on the sphere). Since $\phi=\omega t$,

$$
\frac{d^{k}}{d t^{k}} Y^{\ell m}\left(\frac{\pi}{2}, \phi\right)=(-i m \omega)^{k} Y^{\ell m}\left(\frac{\pi}{2}, \phi\right)
$$

Therefore, it can be shown that

$$
x_{L-2} v_{i_{\ell-1} i_{\ell}} \mathcal{Y}_{L}^{\ell m}=\frac{\ell-m^{2}}{\ell(\ell-1)} \omega^{2} r^{\ell} Y^{\ell m}\left(\frac{\pi}{2}, \phi\right),
$$

using that $\dot{r}$ and $\dot{\omega}$ are of 2.5 order. Finally, using that

$$
\left(\frac{r \omega}{c}\right)^{\ell}=x^{\ell / 2}\left[1-x \ell\left(1-\frac{1}{3} \nu\right)\right]
$$

it can be shown that

$$
\begin{aligned}
h^{\ell m}= & (i m)^{\ell} \frac{8 \pi}{(2 \ell+1) ! !} \sqrt{\frac{(\ell+1)(\ell+2)}{\ell(\ell-1)}} \frac{G \nu \tilde{m}}{c^{2} R} x^{\ell / 2} Y^{\ell,-m}\left(\frac{\pi}{2}, \phi\right)\left\{f_{\ell-1}(\nu)\left[1-x \ell\left(1-\frac{1}{3} \nu\right)\right]+\frac{3}{2} x f_{\ell+1}(\nu)\right. \\
& \left.-x f_{\ell}(\nu)-x \frac{m^{2}(\ell+9)}{2(\ell+1)(2 \ell+3)} f_{\ell+1}(\nu)+O\left(\epsilon^{3 / 2}\right)\right\} \quad \text { (for } \ell+m \text { even) },
\end{aligned}
$$

where $\tilde{m}$ and $f_{k}(\nu)$ are given by (A6).

\section{2. $\quad \ell+m$ odd}

For odd $\ell+m$ (for nonspinning binaries), the spinweighted spherical harmonic components are due to contributions from the radiative current multipoles. To 0PN order, the radiative moments are given simply $\ell$ time derivatives of the source moments. For arbitrary $\ell$, the current moment is given by Eq. (B2). In evaluating the spin-weighted spherical harmonic components from the source current moments, the following identity is useful:

$$
\delta_{z i_{\ell}} n_{L-1} \mathcal{Y}_{L}^{\ell m}=\frac{1}{\ell} \sqrt{\frac{(2 \ell+1)\left(\ell^{2}-m^{2}\right)}{2 \ell-1}} Y^{\ell-1, m}\left(\frac{\pi}{2}, \phi\right)
$$

Following the same steps as above, it can be shown that 
$h^{\ell m}=-(i m)^{\ell} \frac{16 \pi i}{(2 \ell+1) ! !} \sqrt{\frac{(2 \ell+1)(\ell+2)\left(\ell^{2}-m^{2}\right)}{\ell(2 \ell-1)(\ell+1)(\ell-1)}} \frac{G \nu \hat{m}}{c^{2} R} x^{(\ell+1) / 2} Y^{\ell-1,-m}\left(\frac{\pi}{2}, \phi\right)\left[g_{\ell}(\nu)+O(\epsilon)\right] \quad($ for $\ell+m$ odd $)$,

where $\hat{m}$ and $g_{k}(\nu)$ are given by (B3).

[1] B. C. Barish and R. Weiss, Phys. Today 52 (Oct), 44 (1999).

[2] S. J. Waldman, Class. Quantum Grav. 23, S653 (2006).

[3] F. Acernese et al., Class. Quantum Grav. 19, 1421 (2002).

[4] F. Acernese, P. Amico, M. Alshourbagy, F. Antonucci, S. Aoudia, S. Avino, D. Babusci, G. Ballardin, F. Barone, L. Barsotti, et al., Class. Quantum Grav. 23, S635 (2006).

[5] S. Hild, Class. Quantum Grav. 23, S643 (2006).

[6] C. Cutler, T. A. Apostolatos, L. Bildsten, L. S. Finn, E. E. Flanagan, D. Kennefick, D. M. Markovic, A. Ori, E. Poisson, and G. J. Sussman, Phys. Rev. Lett. 70, 2984 (1993).

[7] H. Tagoshi and M. Sasaki, Prog. Theor. Phys. 92, 745 (1994)

[8] A. Królak, K. D. Kokkotas, and G. Schäfer, Phys. Rev. D 52, 2089 (1995).

[9] T. Damour, B. R. Iyer, and B. S. Sathyaprakash, Phys. Rev. D 57, 885 (1998).

[10] L. Blanchet, G. Faye, B. R. Iyer, and B. Joguet, Phys. Rev. D 65 (2002), 71, 129902(E) (2005).

[11] L. Blanchet, T. Damour, G. Esposito-Farèse, and B. R. Iyer, Phys. Rev. Lett. 93, 091101 (2004).

[12] K. Arun, L. Blanchet, B. Iyer, and M. Qusailah, Class. Quantum Grav. 21, 3771 (2004), 22, 3115-3117(E) (2005).

[13] L. E. Kidder, L. Blanchet, and B. R. Iyer, Class. Quantum Grav. 24, 5307 (2007).

[14] F. Pretorius, Phys. Rev. Lett. 95, 121101 (2005).

[15] F. Pretorius, Class. Quant. Grav. 23, S529 (2006).

[16] M. Campanelli, C. O. Lousto, P. Marronetti, and Y. Zlochower, Phys. Rev. Lett. 96, 111101 (2006).

[17] J. G. Baker, J. Centrella, D.-I. Choi, M. Koppitz, and J. van Meter, Phys. Rev. Lett. 96, 111102 (2006).

[18] A. Buonanno, G. B. Cook, and F. Pretorius, Phys. Rev. D 75, 124018 (2007).

[19] E. Berti, V. Cardoso, J. Gonzalez, U. Sperhake, M. Hannam, S. Husa, and B. Brügmann, gr-qc/0703053 (2007).

[20] J. D. Schnittman, A. Buonanno, J. R. van Meter, J. G. Baker, W. D. Boggs, J. Centrella, B. J. Kelly, and S. T. McWilliams (2007), arXiv:0707.0301v1 [gr-qc].

[21] J. G. Baker, J. R. van Meter, S. T. McWilliams, J. Centrella, and B. J. Kelly, gr-qc/0612024 (2006).

[22] M. Hannam, S. Husa, U. Sperhake, B. Brügmann, and J. A. Gonzalez (2007), arXiv:0706.1305v1 [gr-qc].

[23] M. Boyle, D. A. Brown, L. E. Kidder, A. M. Mroué, H. P. Pfeiffer, M. A. Scheel, G. B. Cook, and S. A. Teukolsky (2007), arXiv:0710.0158 [gr-qc].

[24] K. S. Thorne, Rev. Mod. Phys. 52, 299 (1980).
[25] C. Van Den Broeck, Class. Quantum Grav. 23, L51 (2006).

[26] C. Van Den Broeck and A. S. Sengupta, Class. Quantum Grav. 24, 155 (2007).

[27] K. G. Arun, B. R. Iyer, B. S. Sathyaprakash, and S. Sinha, Phys. Rev. D 75, 124002 (2007).

[28] A. M. Sintes and A. Vecchio, in Gravitational Waves: Third Edoardo Amaldi Conference, edited by S. Meshkov (New York, 2000), vol. 523 of American Institute of Physics Conference Series, p. 403, gr-qc/0005059.

[29] A. M. Sintes and A. Vecchio, in Gravitational waves and experimental gravity, edited by J. Tran Thanh Van, J. Dumarchez, S. Reynaud, C. Salomon, S. Thorsett, and J. Y. Vinet (World Publishers, Hanoi, 2000), pp. 73-78, gr-qc/0005058.

[30] T. A. Moore and R. W. Hellings, Phys. Rev. D 65, 062001 (2002).

[31] R. W. Hellings and T. A. Moore, Class. Quantum Grav. 20, 181 (2003).

[32] C. Van Den Broeck and A. S. Sengupta, Class. Quantum Grav. 24, 1089 (2007).

[33] K. G. Arun, B. R. Iyer, B. S. Sathyaprakash, S. Sinha, and C. Van Den Broeck, arXiv:0707.3920v1 [astro-ph] (2007).

[34] M. Trias and A. M. Sintes, arXiv:0707.4434v1 [gr-qc] (2007).

[35] L. Blanchet, Living Rev. Relativity 9, 4 (2006).

[36] L. Blanchet and T. Damour, Philos. Trans. R. Soc. London, Ser. A 320, 379 (1986).

[37] L. Blanchet and T. Damour, Phys. Rev. D 37, 1410 (1988).

[38] L. Blanchet and T. Damour, Phys. Rev. D 46, 4304 (1992).

[39] L. Blanchet, Phys. Rev. D 51, 2559 (1995).

[40] L. Blanchet, Class. Quantum Grav. 15, 89 (1998).

[41] L. Blanchet, Class. Quantum Grav. 15, 113 (1998), 22, 3381(E) (2005).

[42] L. Blanchet, Class. Quantum Grav. 15, 1971 (1998).

[43] L. Blanchet, Phys. Rev. D 54, 1417 (1996).

[44] L. Blanchet, B. R. Iyer, and B. Joguet, Phys. Rev. D 65, 064005 (2002), 71, 129903(E) (2005).

[45] D. Christodoulou, Phys. Rev. Lett. 67, 1486 (1991).

[46] A. G. Wiseman and C. M. Will, Phys. Rev. D 44, 2945 (1991).

[47] K. S. Thorne, Phys. Rev. D 45, 520 (1992).

[48] A. G. Wiseman, Phys. Rev. D 48, 4757 (1993).

[49] L. Blanchet and G. Schäfer, Class. Quant. Grav. 10, 2699 (1993).

[50] P. Jaranowski and G. Schäfer, Phys. Rev. D 57, 7274 (1998), 63, 029902(E) (2001). 
[51] P. Jaranowski and G. Schäfer, Phys. Rev. D 60, 124003 (1999).

[52] T. Damour, P. Jaranowski, and G. Schäfer, Phys. Rev. D 62, 021501 (2000), 63, 029903(E) (2001).

[53] T. Damour, P. Jaranowski, and G. Schäfer, Phys. Rev. D 63, 044021 (2001), 66, 029901(E) (2002).

[54] L. Blanchet and G. Faye, Phys. Lett. A 271, 58 (2000).

[55] L. Blanchet and G. Faye, Phys. Rev. D 63, 062005 (2001).

[56] T. Damour, P. Jaranowski, and G. Schäfer, Phys. Lett. B 513, 147 (2001).

[57] L. Blanchet, T. Damour, and G. Esposito-Farèse, Phys. Rev. D 69, 124007 (2004).

[58] Y. Itoh, T. Futamase, and H. Asada, Phys. Rev. D 63, 064038 (2001).
[59] Y. Itoh and T. Futamase, Phys. Rev. D 68, 121501 (2003).

[60] Y. Itoh, Phys. Rev. D 69 (2004), 064018.

[61] L. Blanchet and B. R. Iyer, Class. and Quantum Grav. 20, 755 (2003).

[62] P. C. Peters, Phys. Rev. 136, B1224 (1964).

[63] L. Blanchet and T. Damour, Ann. Inst. Henri Poincaré, A 50, 377 (1989).

[64] L. Blanchet, T. Damour, and B. R. Iyer, Phys. Rev. D 51, 5360 (1995), 54, 1860(E) (1996).

[65] T. Damour and B. R. Iyer, Ann. Inst. Henri Poincaré, A 54, 115 (1991).

[66] E. Poisson, Phys. Rev. D47, 1497 (1993). 